\title{
Detoxication agents and technologies for animal husbandry under conditions of anthropogenic pollution
}

\author{
Lyubov L. Zakharova, Georgiy A. Zhorov *, Vasiliy I. Dorozhkin, Viktor N. Obryvin, and Natalya A. Brichko
}

All-Russian Research Institute for Veterinary Sanitation, Hygiene and Ecology - Branch of Federal Scientific Center - K.I. Skryabin, Ya.R. Kovalenko All-Russian Research Institute of Experimental Veterinary Medicine, Russian Academy of Sciences, 123022, Moscow, Russia

\begin{abstract}
The impact of human economic activity on the environment is increasingly taking the form of combined pollution with various ecotoxicants including xenobiotics of radiation (caesium-137, strontium90) and chemical (mercury, cadmium, lead and other toxic elements) nature. Today, this is one of the most urgent and insufficiently studied scientific problems. At current levels of systematic intake and accumulation of ecotoxicants in the body of productive animals, various anthropogenic and environmental organopathologies and diseases develop, and the risk of obtaining products that are dangerous to the consumer increases. In this regard, in the conditions of combined technogenic pollution of agroecosystems, it is necessary to solve the problem of ensuring the production of safe and biologically complete livestock products and maintaining the health and useful qualities of farm animals. The article presents the results of research on the development of compositions and technologies for the use of sorption-detoxifying complexes in the form of feed additives to reduce the accumulation and negative effects of ecotoxicants on the animal body and ensure the production of safe products.
\end{abstract}

\section{Introduction}

Scientific and technological progress and intensive development of industrial production are accompanied by an accelerating increase in the range, output value and expansion of chemical compounds application as well as technologies and products containing sources of ionizing radiation. At the same time, the risk of outbrakes of combined anthropogenic pollution with toxic substances of radiation and chemical nature significantly increases.

Farm animals in regions with increased anthropogenic stress are subject to prolonged systematic exposure to technogenic contaminants, which inevitably affects their health, productivity, quality and safety of the products obtained.

Heavy metals with chronic feed intake, as a rule, do not lead to acute poisoning of animals. However, having cumulative properties they negatively affect the functions of many organs and systems of animals. Radionuclides entering the body, depending on tropism, are accumulated in critical organs and tissues and cause prolonged internal irradiation.

Under these conditions, the number of anthropogenic and environmental diseases with a long latent period is increasing. During this period an effective response to the chronic effect of low-intensity contaminants is accumulated $[1,2]$.

Even more dangerous is the combined effect of ecotoxicants of radiation and chemical nature on animal populations and individual biological objects. Their ability to accumulate and worsen the quality of livestock products, cause long-term effects associated with mutagenic, embryotoxic, teratogenic, carcinogenic and immunosuppressive effects.

In addition to the accumulation of ecotoxicants in the organs and tissues of animals and the development of pathologies associated with their influence, the course of such diseases is accompanied by a massive decrease in overall immunological reactivity, inhibition of humoral and cellular mechanisms of natural resistance and other negative processes. The susceptibility of animals to various diseases including pathologies of an infectious and invasive nature increases against this background [3, 4].

In the regions of environmental concern, the main task of livestock producers is to ensure consumer safety. To solve it, various methods of reducing the intake and accumulation of contaminants in animals are used. However, the problem of obtaining livestock products that meet the requirements of modern quality standards and technical regulations remains unresolved until now. Accordingly, the development of special technologies and tools for animal husbandry and veterinary medicine, ensuring the quality and safety of products in areas of anthropogenic pollution, is of crucial economic and social importance.

\section{Materials and methods}

To determine the effectiveness of various sorbents and biologically active substances in the alimentary intake of

* Corresponding author: geo_geo@mail.ru 
ecotoxicants, more than 50 detoxifying agents providing the most significant reduction in the accumulation of toxic elements $(\mathrm{Cd}, \mathrm{Pb})$ and radionuclides $\left({ }^{137} \mathrm{Cs},{ }^{90} \mathrm{Sr}\right)$ in the organs and tissues of animals, both with combined pollution feed, and in the presence of only toxic elements or radionuclides were studied that. Based on the results obtained, more than 30 formulations of sorption-detoxifying complexes were created and tested on laboratory animals.

In our experiments, we used nitrates $\mathrm{Cd}$ and $\mathrm{Pb}$, radionuclides ${ }^{137} \mathrm{Cs}$ and ${ }^{90} \mathrm{Sr}$ at concentrations ten times higher than their permissible levels in feed. Studies were carried out on outbred white male rats with an initial weight of $180-230 \mathrm{~g}$ and rabbits weighing $3.5-4 \mathrm{~kg}$.

Animals received various options of sorptiondetoxifying complexes and ecotoxicants once a day, 7 times a week, for 45-60 seeding days. The daily sorption-detoxifying compositions and ecotoxicants were mixed with ground standard granulated complete feed for laboratory animals and granules were prepared for individual feeding to experimental animals. The rest of the feed dose consisted of feed concentrate taking into account the age-related needs of the animals.

The effectiveness of sorption-detoxifying complexes in experimental animals was assessed by comparison with the results of control groups in which the animals received $\mathrm{Cd}, \mathrm{Pb},{ }^{137} \mathrm{Cs}$ and ${ }^{90} \mathrm{Sr}$ without giving sorptiondetoxifying complexes.

Throughout the experiment, observations were made on the general condition of the animals (appearance, activity, appetite, signs of intoxication, safety). Before the start as well as throughout the experiment, the animals were weighed every 7 days and blood samples were taken.

After the expiration of the seeding period, the animals were killed and samples of organs and tissues (heart, spleen, kidneys, liver, muscles and bones) were taken to determine growth and weight indicators. The content of ${ }^{137} \mathrm{Cs}$ and ${ }^{90} \mathrm{Sr}$ was determined using a beta gamma-ray spectrometer-radiometer MKGB-01 RADEK, $\mathrm{Cd}$ and $\mathrm{Pb}$ were determined by atomic absorption spectrophotometry on an AA240FS apparatus produced by Varian.

In similar experiments on rabbits, the effect of the use of sorption-detoxifying complexes against the background of the combined intake of radionuclides and heavy metals with feed on the organoleptic and physicochemical parameters of meat was studied.

\section{Research results}

To successfully solve the problems of producing safe and high-quality products, livestock breeding technology under the conditions of environmental concern at the first stage should include the following measures:

- assessment of the state of the environment in the agricultural production zone, based on the results of comprehensive chemical-toxicological, radiobiological and sanitary-bacteriological monitoring, which allows to determine the sources of ecotoxicants, the nature and intensity of pollution and to control their content in all links of the trophic chain;

- a comprehensive description of agricultural enterprises located in the zone of environmental risk (state of the feed base and conditions of detention, health status, animal productivity and product quality);

- toxicological assessment of dietary safety based on the totality of present ecotoxicants, whose criterion is the indicators of their maximum allowable content in the daily diet of animals, and the subsequent forecast of livestock production compliance with sanitary standards.

Afterwards, based on the results of integrated monitoring of this region of environmental concern special animal breeding technologies for specific areas of anthropogenic pollution are developed.

Along with the application of a system of organizational, agrotechnical, agrochemical measures that ensure the reduction of the transition of ecotoxicants from soil to feed at the stage of feed production, sorption-detoxification technologies in animal breeding play an extremely important role.

They are based on the principle of efferent therapy, which implies binding and excretion of endo- and exogenous toxicants through the gastrointestinal tract through the use of various sorbents (selective and multifunctional sorbents, specific antidotes, chelates, and others). At the same time, enterosorption is a physiological and easy-to-use method that does not cause serious complications and does not require significant material costs [5-8].

Due to differences in the mechanism of toxic substances, in particular radionuclides and heavy metals, there are practically no universal remedies that fully meet all the requirements. Therefore, with the development of anthropogenic and environmental organopathologies and diseases that accompany and enhance the negative impact of ecotoxicants, the use of only sorbents is insufficient.

In this regard, it is necessary to use a wider range of pharmacological agents, in particular antioxidants, adaptogens, immunocorrectors, vitamin and mineral supplements and other biologically active substances, which compensate for the negative effects that develop in the body as a result of exposure to harmful environmental factors and contribute to the restoration of health and increase the productivity of farm animals and the products quality [9-11].

Thus, the complex use of drugs of multidirectional action in the composition of sorption-detoxifying complexes in the form of feed additives should be considered the most effective, technologically advanced and economically viable method under the conditions of combined feed contamination.

In addition, under the conditions of separate alimentary intake of ecotoxicants the development of 
private technologies for the use of sorption-detoxifying complexes is the most relevant due to the fact that it is the most effective for certain ecotoxicants or their groups and allows to minimize migration and to completely or partially eliminate this or that contaminant in the trophic chain: feed - animal - production.

Considering these factors, certain criteria for the selection of components must be observed to ensure their compatibility, effectiveness and safety in combined use in the process of developing sorption-detoxifying complexes formulations. The most important criteria are as follows:

- the sorption-detoxifying compositions should be developed in relation to a specific ecologically unfavorable region on the basis of the toxicological and radiation monitoring results with regards to the nature and intensity of pollution, the conditions of various branches of animal breeding, data on the survey of the number of animals and analysis of the animal products quality;

- the sorption-detoxifying complexes under development should include both multifunctional and selective detoxicants (complexing agents, ion-exchange substances, mineral or organic sorbents, specific antidotes), and biologically active substances (immunocorrectors, antioxidants, adaptogens, vitamins, micro and macro elements);

- the sorption-detoxifying compositions and their components should provide a reduction in the toxic effects of ecotoxicants on the animal organism and a decrease in their transition from feed to animal organism with an efficiency of more than $50 \%$;

- the sorption-detoxifying compositions and their components should ensure the production of safe products, be compatible and non-toxic to animals, and not adversely affect the quality of the products obtained under conditions of a long period of use while maintaining manufacturability, simplicity and efficiency of their manufacture and use.

Table 1 presents the most important groups of active substances used in the development of sorptiondetoxifying compositions formulations.

Table 1. Main groups of active substances for sorptiondetoxifying compositions development

Groups and brief description of active substances Mineral and organic sorbents, ion-exchange and complexforming substances are a large group of compounds of various chemical structures of natural and synthetic origin and preparations based on them. They have antitoxic properties against a wide range of toxicants of mineral and organic nature, including heavy metals and radioactive substances.

Antioxidant compounds are substances of various chemical nature. They have antioxidant properties, suppress the processes of peroxidation and free radicals formation, have a stabilizing effect on biological membranes, bind and neutralize xenobiotics and endogenous toxins, increase the body's radioresistance.
Immunomodulators (immunocorrectors), having a stimulating effect on the body's immune system, are the compounds of different origin and chemical structure. They combine the stimulating effect and resistance of the body with detoxification, adaptogenic, antioxidant and membraneprotective activity. They contribute to the normalization of energy metabolism, the general physiological state of the body, enhance the processes of biosynthesis in conditions of pathologies and extreme effects.

Feed additives are diet components containing a complex of vital elements, carbohydrates, vitamins and biologically active substances. They reduce the accumulation and negative impact of ecotoxicants on the body, regulate the amount and ratio of nutrients, and ensure the achievement of the highest productivity and preservation of the livestock.

According to the developed technology for the use of sorption-detoxifying complexes depending on the specific conditions (the set of present ecotoxicants, livestock breeding conditions, economic affordability and other influencing factors), the following compomemts can be included in sorption-detoxifying compositions:

- ferrocyanides (ferrocene, bifezh, HG-90, TsIIOM, etc.);

- mineral sorbents (zeolites, bentonite, vermiculite, perlite, etc.);

- carbon sorbents (carbolene, carboktin, gastrosorb, etc.);

- silicon-containing detoxicants (enterosgel, polysurmin, etc.);

- lignin-containing sorbents (polyphepan, pheocarpine, etc.);

- complexing agents (penicillamine, etc.);

- antioxidant compounds (sulfur and its compounds, flavonoids, gallates, carotenoids, tocopherols and other vitamins, selenium, enzymes, etc.);

- natural or modified chitin (chitosan, chitan, chitinmelanin complex);

- humates (ligfol, gumavit, lignohumate, edagum, humadapt);

- immunomodulators that have a stimulating effect on the body's immune system (polyoxidonium, immunofan, roncoleukin, fosprenyl, succinic acid, and other natural and synthetic compounds);

- feed additives (complexes of vitamins and deficient micro and macro elements, amino acids, pre- and probiotics, and other biologically active substances).

In accordance with the developed approaches to the development of sorption-detoxifying compositions for being used in conditions of combined contamination of feed with ecotoxicants of radiation and chemical nature, an approximate list of sorbents and biologically active substances with different bioprotective mechanisms that are promising as sorption-detoxifying components is proposed. Components combinations have been developed and their effectiveness has been studied to reduce the intake of ecotoxicants $\left(\mathrm{Cd}, \mathrm{Pb},{ }^{137} \mathrm{Cs}\right.$ and ${ }^{90} \mathrm{Sr}$ ).

Doses of drugs are calculated based on the weight of an animal, per $1 \mathrm{~kg}$ of live weight or per head per day, per $1 \mathrm{~kg}$ of diet or per diet as a whole. Medications should be introduced directly to the feed concentrate in feed rooms 
or sorption-detoxifying combinations mixed with feed should be preliminarily produced taking into account the compatibility and physico-chemical properties of their active substances.

Sorption-detoxifying complexes are given to animals regardless of the time of day or season, both in stable and outdoor keeping. In cases where oral use of individual components is not possible, they are administered parenterally.

Due to the safety of most components, the period of sorption-detoxifying complexes application can be from 2-3 months to several years, up to the entire period of animals productive exploitation (cows, young cattle older than 12 months, as well as other types of farm animals, taking into account their physiological species features) productive exploitation.

In order to ensure maximum body detoxification efficiency and safety of the products obtained, special attention should be paid to dairy cattle and fattening animals in the pre-slaughter period (2-3 months before slaughter).

The effectiveness of the measures must be monitored by conducting periodic (at least once a quarter) comprehensive studies of animal population state and the quality of the products obtained, according to the results of which it is possible to make the necessary adjustments to sorption-detoxifying complexes and the technology for their application.

Table 2 presents several combinations of sorptiondetoxifying complexes.

Table 2. Sorption-detoxifying complexes most effective in combination with $\mathrm{Cd}, \mathrm{Pb},{ }^{137} \mathrm{Cs},{ }^{90} \mathrm{Sr}$ and feed

\begin{tabular}{|c|l|c|}
\hline No. & \multicolumn{1}{|c|}{$\begin{array}{c}\text { Sorption-detoxifying } \\
\text { combinations }\end{array}$} & $\begin{array}{c}\text { Components } \\
\text { content, wt. \% }\end{array}$ \\
\hline \multirow{4}{*}{1} & Ferrocyanide iron & $1.0-2.0$ \\
\cline { 2 - 3 } & Feed sulfur & $2.0-4.0$ \\
\cline { 2 - 3 } & Zeolite (Irlit-1) & The left part \\
\hline \multirow{4}{*}{2} & Ferrocin & $0.10-0.15$ \\
\cline { 2 - 3 } & Vermiculite & $15.00-20.00$ \\
\cline { 2 - 3 } & Sodium Thiosulfate & $0.05-0.10$ \\
\cline { 2 - 3 } & Sodium Lignohumate & $0.40-0.60$ \\
\cline { 2 - 3 } & Compound feed & The left part \\
\hline \multirow{4}{*}{3} & Ferrocin & $0.10-0.15$ \\
\cline { 2 - 3 } & Vermiculite & $15.00-20.00$ \\
\cline { 2 - 3 } & Sodium Thiosulfate & $0.4-0.5$ \\
\cline { 2 - 3 } & Polysurmin & $0.5-0.7$ \\
\cline { 2 - 3 } & Compound feed & The left part \\
\hline
\end{tabular}

During the studies it was found that the effectiveness of various sorption-detoxifying combinations with respect to ecotoxicants was as follows: cesium-137 was equal to 76-93\%; strontium-90 to 50-79 \%; cadmium to 45-82\%; lead to 59-93\%.

The safety of use and the positive effect on animals' health are confirmed by the results of clinical and biochemical blood tests and calculations of growthweight indicators of animals, organs and tissues.

In experiments on rabbits, it was found that the use of sorption-detoxifying complexes against the background of the combined intake of radionuclides and heavy metals with food provides meat meeting the organoleptic and physico-chemical parameters of healthy animals.

Based on the research results, "Technologies for the use of sorption-detoxifying complexes to reduce the negative impact of xenobiotics of a radiation and chemical nature on the animal organism" was developed (Approved by the zootechnics and veterinary section of the Department of Agricultural Sciences of the Russian Academy of Sciences on 10.10.2019); patents were obtained for individual sorption-detoxifying combinations [12, 13]. 


\section{Conclusion}

To ensure the production of safe products and preserve the health of farm animals in areas with increased anthropogenic stress, it is necessary to use special sorption-detoxification technologies, which are an integral part of a single livestock breeding system in such regions. In this case, the most appropriate way to reduce the accumulation of radioactive substances and toxic elements in the animal body and in the resulting products is the combined use of drugs of multidirectional action and sorption-detoxifying complexes. Their development should be based on compliance with a system of certain criteria that ensure components compatibility, effectiveness and safety of the resulting comibinations.

The effectiveness of sorption-detoxifying complexes developed in the course of research in relation to ecotoxicants is as follws: cesium-137 is equal to 76-93\%; strontium-90 to 50-79\%; cadmium to 45-82\%; lead to 59-93\%, which improves livestock products safety.

The use of sorption-detoxifying complexes against the background of the combined intake of radionuclides and heavy metals with feed into the body of rabbits ensures the production of meat according to the organoleptic and physico-chemical parameters of healthy animals' meat and at the same time helps to restore the physiological parameters of the body.

\section{References}

1. V.P. Aturova, V.A. Domarenko, L.P. Rikhvanov et al., Radioecological problems of the Yenisei river basin, in: Mater. of the Int. sci.-pract. conf. "Radioecology of the XXI century", pp. 194-200 (Siber. Fed. Univer., Krasnoyarsk, 2012)

2. M.V. Kalmykov, Yu.Ya. Mikhailov, Radioactive pollution of the environment, Veter. Pathol., 3, 6-16 (2002)

3. I.M. Donnik, I.A. Shkuratova, N.A. Vereshchak et al., Methodological approaches to assessing the environmental impact on animal health, Agricult. Sci. of the Euro-North-East, 8, 169-173 (2006)

4. A.V. Ospischev, A.S. Kashin, G.V. Kashina, Integrated system of detoxification prophylaxis and pharmacological correction in anthropogenic and environmental diseases of calves, Bull. of the
Krasnoyarsk State Agrar. Univer., 2, 176-181 (2012)

5. N.I. Sanzharova, A.N. Ratnikov, S.I. Spiridonov et al., Technological methods that increase the stability of agrocenoses, restore disturbed lands, optimize farming and obtain agricultural products that meet regulatory standards by producers of various specializations (VNIISHRAE, Obninsk, 2010), $180 \mathrm{p}$.

6. Yu.P. Fomichev, Sorption-detoxification technologies in animal husbandry and veterinary medicine, Agrarian Russia, 5, 3-7 (2004)

7. A.M. Ezhkova, A.Kh. Yapparov, I.A. Yapparov, V.O. Ezhkov, Correction of the content of salts of heavy metals with bentonites in the system "soil plant - animal - livestock products" in regions of varying degrees of technogenic load (Center for Innovat. Technol., Kazan, 2008), 339 p.

8. A.M. Shadrin, Natural zeolites of Siberia in animal husbandry, veterinary medicine and environmental protection (Novosibirsk, 2004), $116 \mathrm{p}$.

9. R.U. Biktashev, S.R. Bulankova, The initiation of metallothionein synthesis against the background of heavy metals rations contamination, in: Mater. of the Int. sci.-pract. Conf. "Actual problems of modern veterinary science and practice", dedicated to the 70 years' anniversary of Krasnodar NIVI, pp. 12-15 (Krasnodar, 2016)

10. I.V. Kireev, T.S. Denisenko, V.A. Orobets, V.A. Belyaev, Oxidative stress in animals and ways of its pharmacological correction, in: Mater. of the Int. sci.-pract. Conf. "Actual problems of modern veterinary science and practice", dedicated to the 70 years' anniversary of Krasnodar NIVI, pp. 182-186 (Krasnodar, 2016)

11. G.V. Konyukhov, K.Kh. Papunidi, R.N. Nizamov et al., Methodological recommendations for the treatment and prevention of combined animal injuries by ionizing radiation, mycotoxins and chemical agents (Approved by the Department of Agricultural Sciences of the Russian Academy of Sciences on 10.25.2018) (Moscow, 2018)

12. V.I. Ignatkin, L.L. Zakharova, P.N Rubchenkov et al., Feed additive for animals, RF patent for the invention no. RU 2374896 C1. (2009)

13. A.M. Smirnov, L.L. Zakharova, P.N. Rubchenkov et al., Feed additive for animals, RF patent for the invention No. RU 2536625 C1. (2014) 